\title{
Faculty Motivators and De-motivators for Teaching Online: Results of Focus Group Interviews at One University
}

\author{
Starr Roxanne Hiltz \\ Information Systems Department \\ New Jersey Institute of Technology \\ roxanne.hiltz@njit.edu
}

\author{
Eunhee Kim \\ Management Information Systems \\ Department \\ Northern State University \\ ekim@northern.edu
}

\author{
Peter Shea \\ Educational Theory and Practice \\ Department \\ University at Albany \\ State University of New York \\ PShea@uamail.albany.edu
}

\begin{abstract}
What are the most significant factors that motivate and inhibit faculty with regard to teaching in online environments? And what are the specific kinds of experiences that underlie and explain the importance of these factors? Exploring these questions through structured focus group discussions is the primary goal of this study. The second goal is to provide insight regarding how to engage and retain a larger number of faculty in online teaching. This paper describes the methods and results of a pilot study conducted using focus group interviews of faculty experienced in teaching using "Asynchronous Learning Networks" (ALN) at one university. Leading motivators are the flexibility allowed by being able to teach "anytime/anywhere;" better/more personal interaction and community building supported by the medium; the technical and creativity challenges offered by this mode of teaching; being able to reach more (and more diverse) students; and better course management. Major sources of dissatisfaction are more work, medium limitations, lack of adequate support and policies for teaching online, and the fact that the medium is not a good fit for some students.
\end{abstract}

\section{Introduction}

Online learning environments provide an unprecedented opportunity to increase student access to higher education, especially for the now- majority of college students who are over 25 and/or balancing education needs with the demands of a job or family. Accomplishing this much needed goal requires the active participation and cooperation not just of the "pioneers" who initially volunteered to try this new mode of teaching but from the majority of university faculty, from a broad spectrum of institutional settings [1].

Although online learning has seen rapid growth in recent years, with estimates of more than 2.5 million higher-education enrollments in online courses in the United States [2], it continues to reflect a relatively small percentage of the entire curriculum of higher education today. We need more systematic evidence to fully explain why online teaching has been successfully adopted at many institutions and to gain insight into why it has not thrived at others.

A productive place to begin this investigation is to examine the factors that both enable and constrain faculty success and satisfaction with online teaching. "Motivators" are defined as factors that increase the desire to teach online, by encouraging, enabling and providing satisfaction, support and rewards (intrinsic or extrinsic). "De-motivators" by contrast, decrease the desire to teach online, by discouraging, constraining, providing sources of dissatisfaction, and decreasing the rewards/effort ratio. "Motivation" and "satisfaction" will be used essentially as synonyms in this paper.

Dedicated, experienced faculty who remain not only willing but also enthusiastic about teaching online are necessary ingredients for a steadily growing and successful online program; yet, anecdotes abound of "burnout" among online faculty. This paper presents the methods and preliminary findings from focus group interviews of experienced online faculty conducted at a medium sized Eastern U.S. university in the Spring of 2006. The total study will include both online surveys of faculty and focus group interviews at two universities. Subsequently, we hope to include a wider variety of institutions of various sizes, types, and geographical locations more representative of higher education in the United States.

The particular form of online learning which is used at the institution studied is Asynchronous Learning 
Networks (ALN), a major type of e-learning. "Asynchronous" refers to computer-mediated communication (CMC) systems that allow "anytime" communication via the Internet, using software platforms such as course management systems, computerized conferencing, or bulletin boards that support threaded discussions. "Learning Networks" refer to the social network or community of learners that emerges when students and faculty communicate and work together to build and share knowledge. ALN thus integrates social and technical aspects; it depends upon technologies such as the Internet and the World Wide Web to link together teachers and learners and learning resources, but it is an effective means of learning only when collaborative social/pedagogical processes emerge from the communication that is supported by the technology [3]. Key to this process is the role of the faculty member to structure and facilitate the online interaction.

For focus group study reported in this paper, our research questions are:

1. When faculty are asked to name their top sources of motivation and de-motivation for teaching online, and to discuss them in a group of peers, does a consistent set of factors emerge from different focus groups at the same university?

2. What are the underlying experiences or components of these factors that explain why they are so important?

3. What are the implications of these findings for steps that could be taken to increase the number of faculty for whom online teaching is a satisfying and rewarding experience?

\section{Review of Theoretical Frameworks and the Research Literature}

We have reached a stage in ALN in which the early adopters are, to a large extent, already involved. We need to know more about the factors that lead and sustain faculty to become engaged and enthusiastic. Models of social change and adoption of innovation can help to provide a framework for acquiring this knowledge. A number of relevant change and adoption models exist $[4,5,6,7,8,9,10,11,12]$ and a component of this research will be to identify which of these models is best suited to understanding adoption of online teaching to guide subsequent stages of the study. A promising direction in this regard may be to look at the adoption of online teaching as a process, rather than an event, reflecting Fuller's [7] Stages of Concern Model, and Hall's [10] Concerns Based Adoption Model (CBAM).
Another benefit of an analysis of current theoretical models is the guidance it can offer in the development of the study design. Roger's [12] Diffusion of Innovation Model was applied to the design of the focus group questioning route. It suggests we simultaneously examine characteristics of the individual adopter, the institutional setting, and the technology itself - steps that have not often been taken in research on faculty adoption of online teaching in higher education. It follows that we expect the leading motivators and de-motivators for faculty will differ according to the institutional setting, may be related to the specifics of the software platform and media used, and may also be related to individual characteristics, particularly the status of the faculty member in regard to tenure and promotion. Thus, for the focus group study presented in this paper, we designed the questioning route to begin with each faculty member describing their individual academic status and prior experiences with teaching online, including the software platform(s) used. This research will eventually compare and contrast results for different colleges and universities, to see to what extent the institutional context does shape the results.

Most of the emerging empirical research on ALNs has focused on students, but the assessment of faculty roles and characteristics that influence their satisfaction with ALNs has received limited empirical attention [13].

Several challenges for prospective faculty were identified in a study by Muilenberg and Berge [14], which examined the barriers that instructors encounter when they transition from face-to-face to distance teaching. Responding instructors identified organizational change and administrative support structures as their main concerns, and also identified lack of technical expertise and social interaction difficulties as important problems. These findings were further supported in a more recent study by Alavi and Gallupe [15], who concluded that institutions often underestimate the need for faculty training and support structures, especially for those who are new to online instruction.

Most studies of faculty behavior and attitudes in relation to online learning point out that substantial change in teaching roles must take place in order to be an effective teacher in this environment. In a series of influential papers, Garrison et al. [16] claim that online instructors must reassess their roles in terms of a series of constructs, including "social presence," "teaching presence" and resultant "cognitive presence" in order to build an effective "community of inquiry." Coppola et al. [17] analyzed 20 semi-structured interviews with ALN faculty at a technological university in terms of role changes that occur when they become virtual 
professors. They classify role changes enacted by instructors in ALN settings in terms of cognitive roles, affective roles, and managerial roles. The cognitive role, which relates to mental processes of learning, information storage, and thinking, shifts to one of deeper cognitive complexity. The affective role, which relates to influencing the relationships between students, the instructor, and the classroom atmosphere, requires faculty to find new tools to express emotion, yet most of the faculty interviewed felt that their relationships with students became more intimate. The managerial role, which deals with class and course management, requires greater attention to detail, more structure, and additional student monitoring. Overall, faculty reported a change in their teaching persona, towards more precision in their presentation of materials and instructions, combined with a shift to a more Socratic pedagogy, emphasizing multilogues with students.

There have been a relatively small number of prior empirical studies of faculty satisfaction with teaching online. Almeda and Rose [19] interviewed nine instructors teaching writing courses online at one university and reported that instructors were generally satisfied with their experience. Concerns related to lack of student motivation, difficulties adjusting to asynchronous course delivery, and compensation. In two of the largest study to date, Fredericksen et al. [18] and Shea et al. [20] found very high levels of satisfaction among SUNY Learning Network faculty who responded to an online survey. For instance, Shea et al. [20] found:

- $33 \%$ of the faculty surveyed felt that their online students performed better, and $41 \%$ felt that there was no difference between their online students and their classroom students. Satisfaction with online teaching was higher for those faculty members who assessed their online student performance to be relatively better.

- $51 \%$ felt that student interaction was higher in online courses, $25 \%$ felt there was no difference, and $24 \%$ felt it was lower in online courses.

- $60 \%$ cited interest in on-line teaching and learning as their motivation for teaching online courses, $9 \%$ cited interest in technology/Internet as the motivation. Results indicate that satisfaction with online teaching was much higher for these cited motivations, relative to others.

- $33 \%$ strongly agreed and $64 \%$ agreed that technology had a positive effect on their teaching. Satisfaction with online teaching was higher, the stronger the feeling that technology had a positive effect.

- Only $23 \%$ felt that technical difficulties made online teaching more difficult. For this group, the satisfaction with online teaching was significantly lower relative to the remaining group.

- $58 \%$ of respondents reported more systematic design of instruction in their online courses, $37 \%$ reported no difference and only $6 \%$ reported less systematic design of instruction.

- $85 \%$ felt the experience of designing and teaching an online course would improve their classroom teaching.

At the University of Central Florida (UCF), Hartman et al. [21] surveyed 39 faculty members teaching in totally online courses, mixed mode courses with reduced "seat time" for the face to face portion, and mixed mode with no reduced seat time. The results of the impact evaluation at UCF indicate that faculty feel that their workload increases, with teaching online, along with the quality and amount of interaction with and between students. On the other hand, they are concerned that on-line teaching may not fit into the academy culture. The authors argue that faculty satisfaction and student outcomes are strongly related and that their interaction is the most important outcome. Finally, the authors conclude that faculty satisfaction is both a dependent and independent variable that is nested within colleges, departments, and program areas.

Shea et al. [1] performed a multivariate analysis on survey data from 913 faculty members in 36 New York colleges. The authors found that a significant portion of variance in a composite factor reflecting faculty satisfaction with online teaching could be modeled based on other composite factors reflecting levels of interaction with students, learning that faculty had gained from online teaching, adequate technical support faculty had received, and the discipline in which they taught. Despite a large and diverse sample size and a relatively sophisticated analytical approach, a majority of the variance in online faculty satisfaction among this sample was unexplained. Clearly, additional research is required.

These prior studies and some of the instrumentation served as the basis for development of an online questionnaire which solicited ratings by faculty of the importance to them of various sources of potential satisfaction or dissatisfaction with teaching online at their university, as explained further below. The online questionnaire was used in this study only as a means of providing "common ground" for faculty participating in the focus groups, prior to their discussions. Data collection via the questionnaires was 
not completed until August 2006, and the results have not yet been analyzed.

\section{Method: Development of the Structured Focus Group Technique}

The major component of this study has been to develop procedures and instruments to assess faculty satisfaction and motivation to teach online. Two modes of inquiry have been undertaken at this stage $-\mathrm{a}$ quantitative method involving the development and administration of an online questionnaire which all faculty at the two participating universities were invited to answer (for which the results are not yet analyzed), and a qualitative approach involving the development of interview protocols to be conducted with focus groups.

Guided group discussion methods such as Nominal Group Technique and Focus Groups are especially well suited to uncovering and documenting the "why" behind opinions, and in obtaining much more depth and breadth of analysis from participants than available from individual data collection methods [22, 23, 24]. In Nominal Group Technique, there is a period of individual generation of ideas before they are shared, and also typically, a rank ordering of the importance of various "lists" that participants develop. In focus groups, there is an extensive group discussion of issues that can benefit from complementary insights. The focus group discussions were structured by first making sure that all participants were aware of the full known range of possible motivators and de-motivators; then having each participant write down their "top three," in line with Nominal Group Technique; and then discussing these factors systematically, often using a round-robin approach to make sure everyone had an equal opportunity to participate. The final step was voting, as in Nominal Group Technique. The sessions lasted 2.5 to 3 hours each.

The process which we developed consisted of several steps, the details of which include:

a. Group composition: Each of the groups consisted of 5-7 experienced ALN faculty; some of whom have tried it once and not repeated. All faculty who had taught at least one online course during the previous three years at a mid-sized Eastern research university were contacted and given a list of available meeting times. Purposive sampling procedures were employed to ensure a balanced sample of relatively new and more experienced online instructors and to include instructors in a range of academic disciplines. Participants were given a lunch or light supper prior to the session, and a $\$ 50.00$ gift card for an online bookstore.

b. Participants were asked to first take the online survey, which systematically listed every possible motivator and de-motivator that was found in the literature, and asked them to rate the importance of each factor to them. The questionnaire consists of 65 items. Based on previous studies of faculty satisfaction with teaching online, this questionnaire is an expansion and generalization of instruments previously used at SUNY (e.g., $[18,20]$ ) and extensively tested in prior research for validity of the constructs. The items measuring importance of potential sources of satisfaction or dissatisfaction were presented in labeled sets which included opportunities for professional growth, interest in reaching new student audiences, job security and tenure and promotion factors, collegiality, material incentives, reputation of online teaching, complexity of developing and teaching courses online, technical support issues, time issues, compensation issues, and quality issues.

c. Besides completing the questionnaire, focus group participants were asked to think about all of the potential sources of satisfaction and of dissatisfaction (including factors that may not have been included in the questionnaire) before arrival at the sessions, and to write down their "top three" in each category and bring this to the meeting. This two-step preparation process both gave the focus group participants a common grounding in the range of factors that might be considered, and also assured that they would have their own independent rankings of factors before the focus group discussion began.

d. A questioning route/set of steps was developed for the Focus Group sessions, which consisted of several parts:

i. Following a light lunch or supper and completion of consent forms, self introductions included experience teaching online and software platforms used.

ii. Each faculty member was asked to describe their most important motivator that had not already been mentioned, which was then posted This "nomination" proceeded in a round robin fashion until there were no more "top motivators" that had not already been suggested. Similar concepts were combined, resulting in a composite list of motivators. Discussion of each of these 
in some detail followed, with each faculty member invited to share experiences or reactions related to each of the constructs in turn. Then rank ordering of this list by the participants was accomplished by each participant giving five points to their first choice, 4 to their second, etc., with a maximum of five of the items on the lists able to be given points by each individual. The points were then totaled and reported to produce the rank ordering. The lists generated and the final voting were recorded on large sheets of self-sticking paper placed along the wall in the front of the room, for all to see during the discussion and voting, and as a record of the results.

iii. After a break, the same process was followed for sources of dissatisfaction, de-motivators.

iv. Taking the top reasons for lack of satisfaction, through the focus group discussion an understanding of stakeholders related to these problems was developed, and also actions that could be taken by specific stakeholders to decrease or solve the sources of dissatisfaction with teaching via ALN were explored.

Two recorders were used and transcripts were made and then the results were coded and analyzed.

\section{Results}

There was considerable agreement among the different groups about the top reasons for wanting to teach online, and also the top sources of dissatisfaction and frustration, although there were a few ideas that were unique to one particular group. We will first summarize the rank order of factors in the different groups, and then expand upon the factors that faculty feel most strongly about, by presenting representative quotes from the discussions.

\subsection{Motivators}

Table 1 shows the top ranked motivators using the terms developed by each group itself.

Table 1. Motivators by group

\begin{tabular}{|ll|}
\hline \multicolumn{1}{|c|}{ Group 1 } \\
\hline 1 & Flexible schedule (22) A \\
2 & Personal interaction (20) B
\end{tabular}

\begin{tabular}{|ll|}
3 & Learning community (10) B \\
4 & Pedagogical challenge (8) C \\
5 & Reach more students (7) D \\
6 & Challenge of technology (4) C \\
7 & More effectiveness than face-to-face (2) \\
\hline & \multicolumn{1}{c|}{ Group 2 } \\
\hline 1 & Time/location flexibility (23) A \\
2 & Diverse students (22) D \\
3 & Faculty creativity (18) C \\
4 & Better interaction/quality (17) B \\
5 & Easier record keeping/course management (10) E \\
\hline & $\quad$ Group 3 \\
\hline 1 & Self-scheduling: anytime/anywhere (23) A \\
2 & Learn new technology (22) C \\
3 & Medium (being online) (20) B \\
4 & Better control (19) E \\
5 & Reaching non-traditional students (10) D \\
6 & Mentoring others (5) \\
\hline & $\quad$ Group 4 \\
\hline 1 & Flexibility of schedule (anytime/anywhere) (14) \\
& A \\
1 & Opportunity to work intensively with students \\
3 & (14) B \\
4 & Course management (11) E \\
5 & Meeting students' needs/desires (10) D \\
6 & Diverse community (4) D \\
\hline & \\
&
\end{tabular}

The same procedures were repeated for the demotivators, and Table 4 presents a combined list of demotivators. We noted that several of the different terms are essentially synonyms for the same idea. Thus, all motivators of each group were pooled altogether, and similar ideas were combined by adopting a common terminology to generate a consolidated list of motivators. Letters in the lists in Tables 1 (and 3) show the categories into which differently worded factors that refer essentially to the same idea were combined. Table 2 shows the combined motivators.

\section{Table 2. Combined motivators}

\begin{tabular}{|ll|}
\hline \multicolumn{1}{|c|}{ Leading Motivators } \\
\hline A & Flexible schedules: anytime \& anywhere (82) \\
B & $\begin{array}{l}\text { Better/personal interaction- medium } \\
\text { characteristics improve pedagogy (81) }\end{array}$ \\
C & $\begin{array}{l}\text { Challenge/Creativity/Professional Development } \\
\text { (58) }\end{array}$ \\
D & Reach more diverse students (53) \\
E & Better course management (40)
\end{tabular}

As can be seen, when the results of the four groups are combined, the top motivators are flexible schedules due to fact that teaching can be done "any time, any place" where an Internet connection can be obtained. 
This is followed by aspects having to do with the pedagogical advantages of the medium (e.g., more personal interaction, being able to build a learning community); the challenge and stimulation of learning new technology and creatively developing new pedagogical techniques; reaching more and more diverse students; and improved course management capabilities (largely due to software tools).

Flexible schedules enabled by anytime/anywhere teaching/learning topped the list of motivators. One female professor with extensive administrative as well as teaching responsibilities says, "It enables me to teach and I think that really goes under self scheduling because my calendar is so full and needs to be so flexible that if I were to teach a face-to-face course or more than one face-to-face course, it would be impossible for me to schedule other things that need to be scheduled." Another male faculty member in the Information Systems department says, "For example, one of the courses I teach is only going once a year and if we offered it face-to-face, and say our students are from various places, it very well could conflict with the class, but by offering it online it satisfies all possibilities."

Ability to accommodate family responsibilities also was frequently connected to the advantages of teaching online. For instance, a mother of three young children mentioned, "The ability to move around if you need to, and the other thing is family balance, which is part of the moving. If you're in another location, you're still accessible (to your students)." An older faculty member with elder care responsibilities stated, "It came in very handy for me during my mother's illness a couple of years ago, because I had to be in Florida for a couple weeks." Still another theme related to time and place flexibility is the ability to take advantage of professional opportunities such as attending conferences or spending part or all of a semester abroad. For example, a faculty member mentioned, "Flexibility of location and time. I spent one semester in France; I was teaching here distance learning, so that it was real distance learning!"

Essentially tied as a leading motivator is the perception that pedagogy is improved by unique characteristics of online teaching using asynchronous text based discussion. There are several dimensions to this. Self pacing for the students was mentioned frequently. But so was the ability to interact more, and more personally, with students. For example, one instructor stated, "You begin to learn about the work habits of your students very quickly and I think you get to know them, because you're dealing with them in a verbal way as opposed to a visual way... You don't have these interactions in the classrooms, where mostly I'm doing the talking in front of the classroom and (only) some students will raise their hands, but I won't get to know them better." Another explanation ties together the nature of text based asynchronous communication with greater participation and better faculty knowledge about the students as follows: "In the online classes, I get to know my students better because I spend so much more time with them because when they write, they don't write the way we speak in a class. We speak one sentence, two sentences at the most; but in an online course the students will write for four or five paragraphs, and so you get much, much more input from every student, and you get to know each student much better."

Another aspect is the advantage of written text based communication for students for whom English is not the native language: "In the classroom, if a student has a heavy accent, or somebody is not there, or they mumble, or someone is paying attention to something else at the moment, what they say is lost. Whereas online, there is a transcript of everything that goes on. So, that's what I meant by (stating) that they learn more from each other." Another aspect of the advantages of the medium is it supports the emergence of a learning community among the students: "Students in e-learning have told me that they formed better relationships with other students than they would if they were in a face to face environment. That's kind of a motivator for me because I feel I get closer to students."

The challenges and satisfaction of learning new technologies and creatively applying them to teaching were also frequently mentioned. In particular, it was pointed out that use of technology in online teaching can improve the creativity of instructors. One male faculty member says, "One of the things that I like about teaching online is it encourages the instructor to use their creativity to create interesting things online which we don't always do in the classroom. It's enhancing creativity on the part of the instructor." Another faculty member explained, "I have to think a lot about how to motivate students to catch up with readings and assignments. It was really challenging for me and I really enjoyed it and I find many different ways to motivate them. That was the reason that I really like online teaching. I had to think. It was a big challenge for me to motivate them. It was kind of an experiment for me." Many faculty members also echoed the sentiments of an instructor who described "the challenge of the technology... I just happened to enjoy using new technology that I haven't used before and most of the time they worked well, occasionally they were frustrating, and exploring on how I can use the new technology such like using the WebCT. What new ways can I use it that I haven't used it before? 
Personally, it is interesting and in the classroom, the technology is almost always the same."

That online courses can reach more, and more diverse, students is the fourth biggest motivator. Participants say that increased diversity of students can add more value to online learning. For example, one male faculty member says, "In my class I know there are students who are taking my class while they are working in England or in California or in Oregon. Now these students came from Microsoft. They really add a lot of richness to the discussion. That's a really good quality." Geographic and cultural diversity was also mentioned: "I've had students in Japan, Singapore, and India..." The expansion of opportunity to students who could not otherwise take university courses came up in every group. For example, one faculty member stated, "This way I can reach the students who might not have the opportunity to attend classes but they are very enthusiastic and they really want to learn." As an example, she described "The ladies in my class that were in their $40 \mathrm{~s}$, and one of them was pregnant and she was telling me that she had three other kids. That was the only way that she could attend the classes."

Easier course management is another big motivator. Participants say that the virtual classroom environment can allow more effective and efficient management of course materials and student participation. One female faculty member described the advantages of course management systems, "Easier recordkeeping for the class. Tracking students' participations and having all their assignments in one place, it's easier for them too because they then can see their grades when they're posted and they can keep track of the teachers comments." Another aspect is easier and more efficient distribution of course materials; students cannot lose or misplace the materials, since they can always find them online.

\subsection{De-Motivators}

Table 3 shows the results for each individual focus group, whereas Table 4 combines similar terms and shows the overall rank order that results from combining the ranking results.

\section{Table 3. De-motivators by group}

Group 1

1 More work overall (25) A

2 Lack of visual/direct connection with students (14) B

3 Expectations of higher availability and attention from students (7) A

4 Exam process (5) B

4 Course development (5) A
6 Loneliness from working alone at home (4)

6 Evaluation process (4) E

8 Too many unqualified students are allowed to take DL courses (3) C

$9 \quad$ Some class sizes are too large (2) C

Group 2

1 Lack of face-to-face/synchronous contact (19) B

2 More work/no more pay (18) A

3 Characteristics of DL students (15) D

4 Dropouts/losing students (13)

4 Lower student evaluation (13) E

6 Issues of control (6) B

7 Lack of technology at (university) (5) C

8 DL exam (1)

Group 3

1 Medium problems (28) B

2 Workload (20) A

3 Lack of recognition from

peers/administration/general staff/students (14) F

4 Course evaluation (10) E

5 Training and support at (university) (11) C

6 Lack of compensation/control (7) A

Group 4

1 More work/time (17) A

1 Lack of policy in (university) (17)C

3 Lean text-only medium (14) B

3 Does not fit all students (14) D

5 (University) emphasizes profits over quality (9) C

6 Lack of peer/administration recognition (4) $\mathrm{F}$

Table 4. Combined de-motivators

\begin{tabular}{|ll|}
\hline \multicolumn{1}{|c|}{ Leading De-motivators } \\
\hline A & More work/inadequate compensation (99) \\
B & Medium problems (86) \\
C & Lack of support/appropriate policies for online \\
& teaching (48) \\
D & Does not fit all students (29) \\
E & Ineffective/poor evaluation (27) \\
F & Lack of recognition (18) \\
\hline
\end{tabular}

In terms of de-motivators, two factors stand out: more work and medium problems. Participants perceive that online teaching requires more time and energy than face-to-face teaching. An experienced part time faculty member who has taught the same course both online and face to face for several years summed up her feelings by saying, "I think it's more work, stressful and more time consuming too." A male faculty member says, "It's a lot tougher to teach a distance course, it's a lot more work for the instructor. It forces us to communicate better. And also forces us to create better learning materials. Because just 
attaching the textbook with some videos, or whatever, is not really the norm anymore." Another faculty member describes the constant demand to be online and responsive, a theme repeated by many others: "I had to $\log$ in a couple of times a day, or sometimes more than that. I had to respond to them immediately, otherwise they wouldn't have done their assignments, they would have said, 'Oh, you didn't answer my emails.",

The administrative difficulty of dealing with a barrage of assignments handed in online is also described frequently in terms of adding to the workload. "Opening up all those files and trying to organize them. I have to open it up, read through and grade it, make sure that the grade gets recorded in my spreadsheet, move that email into a separate folder so that I have a record of it, and reply to the student."

Regarding medium problems, participants pointed out that they are highly related to the fact that the major communications method in online teaching is based on text. One female participant says, "I'm going to bring one up that's different but what you're really talking about again, it has to do with the medium, is that I think it really is because it's a text based, lean medium and you lose a lot of emotional cues."

Interestingly enough, it was also pointed that more work in online teaching is mostly caused by media problems, implying that solutions to media problems may reduce workload of instructors teaching online. For example, one participant says, "I think workload for me is related to media problems. Inextricably, of all the things that I said and other people said about the medium,... it's text based, if we're going to another modality and that's the fix, that's not solely text based, I think you'll change [decrease] the workload a lot."

Lack of appropriate institutional support and policies for online teaching ranks third. Many participants said that they feel lack of administrative and technical support discourage online teaching. For example, one participant states, "Well, it's hard to argue that if you have to do more with fewer resources. It is a problem that's daunting because we know in fact the kinds of technology that would make this a much richer kind of thing, all of which cost money which we don't have."

At the institution studied, in recent years, the average student teaching ratings for online courses have tended to be lower than the average for face-toface courses. This harms the faculty in terms of promotion, tenure, merit raises, and recognition. There was considerable speculation about the causes of this phenomenon in the groups. One reason suggested was that face-to-face evaluations are given in class and thus have a very high response rate. For online course evaluations, students have to choose to go to the web site and complete the questionnaire on their "own" time rather than during class time. Response rates are much lower. Several faculty suggested that the most disgruntled students are the most likely to take the time to complete these evaluations, just as it seems that a disproportionate number of unhappy students use optional teacher rating sites such as "RateMyProfessors.com." In another group where the selection bias in who completes the ratings for online professors came up, one professor expanded on the idea of selection bias: "I also think that it's some selection bias not only in responses, but in [who is] taking the course, they're probably too busy, they have to do something else and their commitment to the expectations of the course are that this is going to be easier, then they find out it's not."

Others speculated that both some faculty and some students have incorrect assumptions about what it takes in terms of effort to successfully teach and learn online. For example, a woman who had both taught online and been a student in graduate courses said, "You know a lot of instructors, they teach DL because they think it's easier to teach, and at the same time a lot of student take it because they think it's easier to study that way, both of them are wrong." A senior faculty member chimed in, "And I think that accounts for some of the lower evaluations of DL faculty." A third said she had talked to some of the students who had not done well and who seemed dissatisfied, and they said, "Oh, you were giving us a lot of work, we didn't' expect to do that much in a DL course, its just so much work."

Another important de-motivator is that the unique characteristics of online learning may not fit some students. One male participant says, "The older ones are uneasy with discussion. They come in (to the class) with, you know, young people grew up with computers, so it's like swimming to them, but older people... sometimes their skills are dated.. But if they've been working in a police department, or have been working as a reporter, for instance, they sometimes have skills. So there are people with no skills, and people with super skills at the same time" (and teaching to both in the same class can be difficult.)

Several faculty spoke very passionately about the fact that they felt that they worked harder (and did a better job) online, but were actually devalued or stigmatized by the administration and their peers for teaching online. For example, one instructor lamented, "I have a huge problem about lack of recognition from the community.... Lack of recognition from my peers - all of my buddies in my school of management, they all laugh at me, because I don't go to the class. As if I'm not doing anything. So I wonder whether the administrators also think that way, that we are getting 
away with nothing... distance learning takes more time than face to face. But I don't worry about it, that's ok with me. The point is that people all laugh at me, they think that you are very lazy." Another faculty member in the group jumped in saying, "Not only peers and administration, I think there's a general stigma like "mail order degree" kind of thing."

\section{Discussion and Conclusion}

The combination of nominal group technique and focus group discussion that we used has the advantage of generating evidence about why and how various motivators and de-motivators are important to faculty. Just as online discussions can be synergistic, the group discussion stimulated faculty to think of examples and extensions of ideas that they otherwise would not have thought of. It was evident during the discussions that the discussion process itself changed the thinking of some participants about what were the most important sources of satisfaction and dissatisfaction. Though not reported here, the discussion of steps that could be taken to alleviate some of the de-motivators also benefited from the group interaction. Thus, the method is recommended to other institutions that wish to obtain a better understanding of their online faculty and of steps that can be taken to improve their motivation for teaching online. However, there are two important caveats. One is that because faculty need to devote a half day to preparing for and participating in this process, they need to be compensated in some form. Thus, one needs a budget to cover these expenses. The second is that transcribing the focus group recordings is quite time consuming, especially when one wants to identify the various speakers so that their opinions can be related to their experience and status and characteristics. It took between 8 and 16 hours per transcript for this study, for each 2.5- 3 hour recording.

Though the results of separate groups' rankings were combined in this paper to produce an overall ranking, the numbers produced should be interpreted in a qualitative rather than a quantitative way, meaning that a different set of groups would undoubtedly produce different "numbers." However, the striking similarities in top motivators and de-motivators among all four groups indicate that the overall results are valid for this one institution.

Among the most actionable results that we obtained is the following puzzle: faculty say that they work harder online, and that they think that most of the students they teach learn more via this medium, and that they are able to reach more students and a greater diversity of students. However, many feel that their efforts are not only not rewarded, but actually devalued by the institution and by many of their colleagues. They lament the absence of policies that adequately train, guide, and reward online faculty. Certainly, the institution at which this study took place could take steps to change this situation. Official praise and encouragement from high administrative levels, which costs nothing, would go a long way towards eliminating this source of dissatisfaction.

The most interesting methodological results of this study will come from comparing the statistics gathered from the online survey taken by a much larger number of faculty, with the outcomes of the focus groups. We expect that they will be complementary. Secondly, we expect that the results for the two initial universities studied will be different in substantial ways and similar in others; however, as we do not have the two sets of outcomes available for this paper, this remains a speculation. If they are different, this will add support to theories such as those by Rogers [11] about the importance of contextual factors in modifying the impacts and effects of a technology such as ALN. In terms of practical implications of the results, we need to determine generalizability beyond these first two institutions, to a much broader set of institutions, ideally outside of the U.S. as well as inside.

By understanding faculty motivations related to teaching online, we hope to generate recommendations that will help to engage and retain a larger number of online instructors. Ultimately the objective of increasing access to quality higher education for a far larger number of students, many of whom cannot easily attend traditional courses, will thereby be advanced.

\section{Acknowledgments}

This research is partially supported by the Alfred P. Sloan Foundation. Students Chike Uzoka and Adonis Peralta and the second author assisted with transcribing the focus group discussions. We are grateful to the many faculty members who made time in their busy schedules to participate in this study.

\section{References}

[1] P. Shea, A. Pickett, and C. Li, "Increasing access to higher education: A study of the diffusion of online teaching among 913 college faculty", International Review of Research in Open and Distance Learning, 6 (2), 2005.

[2] E.I. Allen and J. Seaman, Entering the Mainstream: The Quality and Extent of Online Education in the United State, The Sloan Consortium, 2003 and 2004. 
[3] S.R. Hiltz and R. Goldman, eds, Learning Together Online: Research on Asynchronous Learning Networks, Erlbaum, Mahwah, NJ, 2005.

[4] S.E. Anderson, "Understanding teacher change: Revisiting the concerns-based adoption model", Curriculum Inquiry, 27, pp. 331-367.

[5] D. Cheung, J. Nattie, and N. Davis, "Re-examining the stages of concern questionnaire: A test of alternative models", The Journal of Educational Research, 94, 2001, pp. 226-236.

[6] E. Davis, R. Bagozzi, and P. Warshaw, "User acceptance of computer technology: A comparison of two theoretical models", Management Science, 35 (8), 1999, pp. 982-1003.

[7] F.F. Fuller, "Concerns of teachers: A developmental conceptualization", American Educational Research Journal, $6(2), 1969$, pp. 207-226.

[8] G.E. Hall, R.C. Wallace, and W.A. Dossett, $A$ developmental conceptualization of the adoption process within educational institutions, Austin, Research and Development Center for Teacher Education, University of Texas at Austin, 1973.

[9] G.E. Hall and S.M. Hord, Change in schools: Facilitating the process, State University of New York Press, Albany, NY, 1987.

[10] G.E. Hall and S.M. Hord, Implementing Change: Patterns, principles, and potholes, Allyn \& Bacon, Needham Heights, MA, 2001.

[11] E.M. Rogers, Diffusions of Innovations, First Edition, Free Press, New York, 1963.

[12] E.M. Rogers, Diffusions of Innovations, Fifth Edition, Free Press, New York, 2003.

[13] C. Dzuiban, P. Shea, and J.B. Arbaugh, Faculty Roles and Satisfaction in Asynchronous Learning Networks, In S.R. Hiltz and R. Goldman, eds, Learning Together Online: Research on Asynchronous Learning Networks, Erlbaum, Mahwah, NJ, 2005, pp. 169-190.

[14] L.Y. Muilenburg and Z.L. Berge, "Barriers to distance education: A factor-analytic study", The American Journal of Distance Education, 15 (2), 2001, pp. 7-22.

[15] M. Alavi and R.B. Gallupe, "Using information technology in learning: Case studies in business and management education programs", Academy of Management Learning and Education, 2 (2), 2003, pp. 139-153.

[16] D.D. Garrison, T. Anderson, and W. Archer, "Critical thinking, cognitive presence and computer conferencing in distance education", The American Journal of Distance Education, 15 (1), 2001, pp. 7-21.
[17] N.W. Coppola, S.R. Hiltz, and N.B. Rotter, "Becoming a virtual professor: Pedagogical roles and asynchronous learning networks", Journal of Management Information Systems, 18(4), 2002, pp. 169-189.

[18] E. Fredericksen, A. Pickett, P. Shea, W. Pelz, and K. Swan, "Factors influencing faculty satisfaction with asynchronous teaching and learning in the SUNY Learning Network", Journal of Asynchronous Learning Network, 4 (3), 2000 .

[19] M.B. Almeda and K. Rose, "Instructor Satisfaction in University of California Extension's On-line Writing Curriculum", Journal of Asynchronous Learning Network, 4 (3), 2000.

[20] P. Shea, W. Pelz, E. Fredericksen, and A. Pickett, Online teaching as a catalyst for classroom-based instructional transformation, Elements of Quality Online Education, SCOLE, Needham, MA, 2002.

[21] J. Hartman, C, Dziuban, and P. Moskal, "Faculty Satisfaction in ALNs: A Dependent or Independent Variable?", Journal of Asynchronous Learning Network, 4 (3), 2000.

[22] J. Nielsen, Use and misuse of focus groups, www.useit.com/papers/focusgroups.html, $\quad$ Retrieved November 2005.

[23] R. Krueger, Focus Groups: A practical guide for applied research, Second Edition, Sage, Thousand Oaks, CA, 1994.

[24] S. Hesse-Biber and P. Leavy, The Practice of Qualitative Research, Sage, Thousand Oaks, CA, 2006. 